\title{
What Kinds of Posterior Cruciate Ligament Bundles Are Preserved in Cruciate-Retaining Total Knee Arthroplasty? A Three-Dimensional Morphology Study
}

\author{
Yoshinori Inou, MSc ${ }^{1}$ Tetsuya Tomita, MD, $\mathrm{PhD}^{1}$ \\ Kazuomi Sugamoto, MD, PhD ${ }^{1}$ \\ ${ }^{1}$ Department of Orthopaedic Biomaterial Science, Osaka University \\ Graduate School of Medicine, Suita, Osaka, Japan \\ 2 Department of Orthopaedic Surgery, Osaka University Graduate \\ School of Medicine, Suita, Osaka, Japan
}

Dai Kiyotomo, $\mathrm{MSc}^{1}$ Hideki Yoshikawa, MD, PhD²

\begin{abstract}
Address for correspondence Tetsuya Tomita, MD, PhD, Department of Orthopaedic Biomaterial Science, Osaka University Graduate School of Medicine, 2-2 Yamada-oka, Suita Osaka 565-0871, Japan (e-mail: tomita@ort.med.osaka-u.ac.jp).
\end{abstract}

\begin{abstract}
Keywords

- PCL attachment

- posterior cruciate ligament

- posterior slope

- tibial cut

- total knee arthroplasty

In this study, the effects of tibial bone cutting on the attachment area of the posterior cruciate ligament $(\mathrm{PCL})$, including both the anterolateral bundle (ALB) and posteromedial bundle (PMB), in cruciate-retaining total knee arthroplasty (CR-TKA) were simulated using a three-dimensional (3D) model of the tibial reconstruction. A total of 40 knees with medial osteoarthritis in patients scheduled for TKA were evaluated. Following surface registration of 3D computed tomography (CT) and 3D magnetic resonance imaging (MRI) models of the tibia with the $\mathrm{PCL}$, the area of $\mathrm{PCL}$ attachment was calculated. Tibial bone cutting in the 3D CT model was simulated at 8 and $10 \mathrm{~mm}$ distal to the center of the lateral articular surfaces of the tibia and with posterior tibial slope angles of 0,7 , and 10 degrees. The percentages of the ALB and PMB tibial attachment areas that remained after cutting at $8 \mathrm{~mm}$ were, respectively, $21.8 \pm 19.7 \%$ and $86.3 \pm 17.7 \%$ with 0 degrees, $9.5 \pm 10.4 \%$ and $64.1 \pm 23.6 \%$ with 7 degrees, and $8.1 \pm 6.7 \%$ and $51 \pm 25.3 \%$ with 10 degrees. These results suggest that only half of the $\mathrm{PCL}$ attachment area was left after the bone cut simulation. Complete preservation of the ALB and PMB attachment areas requires appropriate selection of surgical procedures and devices.
\end{abstract}

Cruciate-retaining total knee arthroplasty (CR-TKA) offers excellent clinical outcomes in the treatment of end-stage knee joint damage. In this surgery, the posterior cruciate ligament (PCL) may be preserved. This ligament functions to stabilize the knee in the anteroposterior direction, resulting in rollback during deep flexion after CR-TKA. ${ }^{1-4}$ The procedure also preserves the proprioception of the ligament, which has been known as a somatic sense for detecting position and motion of the knee joint. ${ }^{5,6}$ However, some reports have shown that as compared with posterior-stabilized TKA, CRTKA decreases the range of motion, with less reproducibility of

received

May 7, 2018

accepted after revision

September 7, 2018

published online

November 10, 2018 rollback in deep flexion. ${ }^{7,8}$ These results suggest that the preserved PCL is not always functional after CR-TKA.

In CR-TKA, the tibial attachment of the PCL is recommended to be preserved on a bone island, but in actual clinical settings, this procedure risks fracture of the bone island. ${ }^{9}$ As a result, many surgeons prefer complete resection of the proximal tibia without a bone island for tibial attachment of the PCL.

Anatomically, the PCL contains two types of ligament fibers, the anterolateral bundle (ALB) and the posteromedial bundle (PMB). The ALB attaches mostly to the roof of the intercondylar notch, whereas the PMB attaches mostly to the
Copyright $\odot 2019$ by Thieme Medical Publishers, Inc., 333 Seventh Avenue, New York, NY 10001, USA. Tel: +1(212) 584-4662.
DOI https://doi.org/ 10.1055/s-0038-1675184. ISSN 1538-8506. 
Inou et al.

medial side wall of the notch on to the medial femoral condyle. ${ }^{10}$ Their movements thus vary depending on the position of the knee joint. ${ }^{10}$ The tibial PCL attachment is close to the articular surfaces. ${ }^{11-14}$ The ALB attachment area is located close to the tibial plateau surface, whereas the PMB attachment area extends below the ALB attachment area. The ALB attachment area or both the ALB and PMB attachment areas may therefore be partly resected depending on the amount of the tibial cut and the posterior tibial slope angle. In fact, previous reports using radiography or magnetic resonance imaging (MRI) after CR-TKA appear to have demonstrated partial resection of the PCL attachment area. ${ }^{15,16}$ These studies used two-dimensional images in the sagittal plane. However, the PCL attachment area is three-dimensional (3D), and 3D evaluations of the ALB and PMB attachment areas before the proximal tibial bone cut are certainly useful to surgeons. Furthermore, the ALB and PMB have different functions and should be investigated separately. The recent development of various image analysis techniques has allowed visualization of the features of the attachment areas of the PCL, ALB, and PMB. ${ }^{12}$ These approaches permit accurate identification of the attachment areas of these ligaments in a 3D bone model.

On the basis of this background, the effects of tibial cuts on the attachment sites of the ALB, PMB, and PCL were investigated in patients with medial knee osteoarthritis $(\mathrm{OA})$ who were scheduled to undergo surgery using a 3D tibial bone model based on computed tomography (CT) and MRI data.

\section{Materials and Methods}

\section{Subjects}

We evaluated 40 knee joints in 40 medial OA patients (12 men and 28 women) who were scheduled to undergo TKA. This study was approved by our Institutional Review Board, and written informed consent was obtained from all the patients.

The mean (range) age, height, weight, and body mass index (BMI) of the patients were 73.3 years (53-86 years),

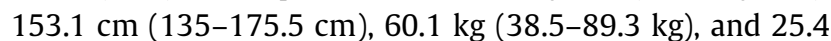
$\mathrm{kg} / \mathrm{m}^{2}\left(17.9-38.3 \mathrm{~kg} / \mathrm{m}^{2}\right)$, respectively. The Kellgren-Lawrence grade for OA was III in 17 knees (42.5\%) and IV in 23 knees (57.5\%; - Table 1).

\section{Methods}

The procedure to identify and measure the PCL attachment area consisted of the following steps.

\section{CT and MRI Acquisition}

CT of the knee joint and ankle joints was performed in the supine and knee extension positions (helical CT, LightSpeed Ultra16, General Electric, Waukesha, WI). The slice thickness was $1.25 \mathrm{~mm} .{ }^{17}$ MRI of the knee joint was performed in the same positions (1.5-T Magnetom Espree, Siemens, Erlangen, Germany; wide gantry diameter: $70 \mathrm{~cm}$; repetition time: 12 ms; echo time: $5.8 \mathrm{~ms}$; flip angle: 20 degrees; field of view: $240 \times 240 \mathrm{~mm}$; matrix: $256 \times 256$ ). The slice thickness was $0.8 \mathrm{~mm}$.
Table 1 Demographic characteristics of the study participants

\begin{tabular}{|c|c|c|}
\hline \multicolumn{2}{|l|}{ Parameter } & \\
\hline \multicolumn{2}{|c|}{ Number of knees } & 40 \\
\hline \multicolumn{2}{|c|}{ Kellgren-Lawrence grade } & III: 17, IV: 23 \\
\hline \multicolumn{2}{|l|}{ Sex } & 12 men and 28 women \\
\hline \multicolumn{2}{|l|}{ Age, y } & $73.3 \pm 6.8(53-86)$ \\
\hline \multicolumn{2}{|l|}{ Height, cm } & $153.1 \pm 9.6(135-175.5)$ \\
\hline \multicolumn{2}{|l|}{ Weight, kg } & $60.1 \pm 12.6(38.5-89.3)$ \\
\hline \multirow[t]{2}{*}{$\begin{array}{l}\text { Preoperative } \\
\text { ROM }\end{array}$} & Flexion & $\begin{array}{l}130 \pm 11.6 \text { degrees } \\
(105-155 \text { degrees })\end{array}$ \\
\hline & Extension & $\begin{array}{l}-10.1 \pm 6.5 \text { degrees } \\
\text { ( }-22 \text { to } 0 \text { degrees })\end{array}$ \\
\hline \multicolumn{2}{|c|}{ Body mass index, $\mathrm{kg} / \mathrm{m}^{2}$} & $25.4 \pm 4.1(17.9-38.3)$ \\
\hline
\end{tabular}

Abbreviation: ROM, range of motion.

\section{Three-Dimensional Bone Model Preparation}

A 3D model was prepared on the basis of the CT and MR images. A 3D Workstation (Volume Analyzer Synapse Vincent, Synapse 3D, Fujifilm, Tokyo, Japan) was used to segment each image. The MR images were segmented for the tibia and PCL. 3D bone models were prepared from the CT and MR images at thresholds of $-2,048$ to 3,238 .

\section{Identification of the PCL Attachment Area}

The PCL attachment area was identified by the first author(Y.I.) who experienced about this procedure using software developed in our laboratory (Orthopaedics Viewer, Osaka, Japan). ${ }^{18}$ Surface registration of the 3D MRI model of the tibia with the PCL superimposed over the 3D CT model of the tibia was applied ( - Fig. 1A)..$^{18,19}$ As the PCL could be visualized in the 3D CT model by superimposition of the 3D MRI model, the outline of the PCL attachment area in the 3D MRI model of the tibia was identified using the 3D CT model. After identification, the 3D MRI model of the tibia with the PCL was deleted, and the outline of the PCL attachment area remained on the 3D CT model of the tibia. The outline of the PCL attachment area was then deleted, and the remaining area was defined as the PCL attachment area. As the ALB and PMB attachment areas have different slope angles, these areas were identified on the basis of the morphology of the PCL attachment area ( - Fig. 1A)..$^{14}$ The ALB and PMB attachment areas were obtained by dividing each area by the total area of the PCL attachment.

\section{Setting the Axis for Tibial Bone Cutting}

Orthopaedics Viewer was used to perform tibial bone cutting in the 3D CT model of the tibia with the identified PCL attachment area. ${ }^{18}$ The vertical axis of the tibia was defined by a line connecting the center of the proximal tibial articular surfaces at the level of the fibular head and the center of the dome of the distal tibial articular surfaces. The anteroposterior axis was defined as a line vertical to a line connecting the proximal posterior tibia/fibula. The 3D CT model of the tibia with the identified PCL area was cut at 8 and $10 \mathrm{~mm}$ distal from the center of the lateral articular surfaces with a 

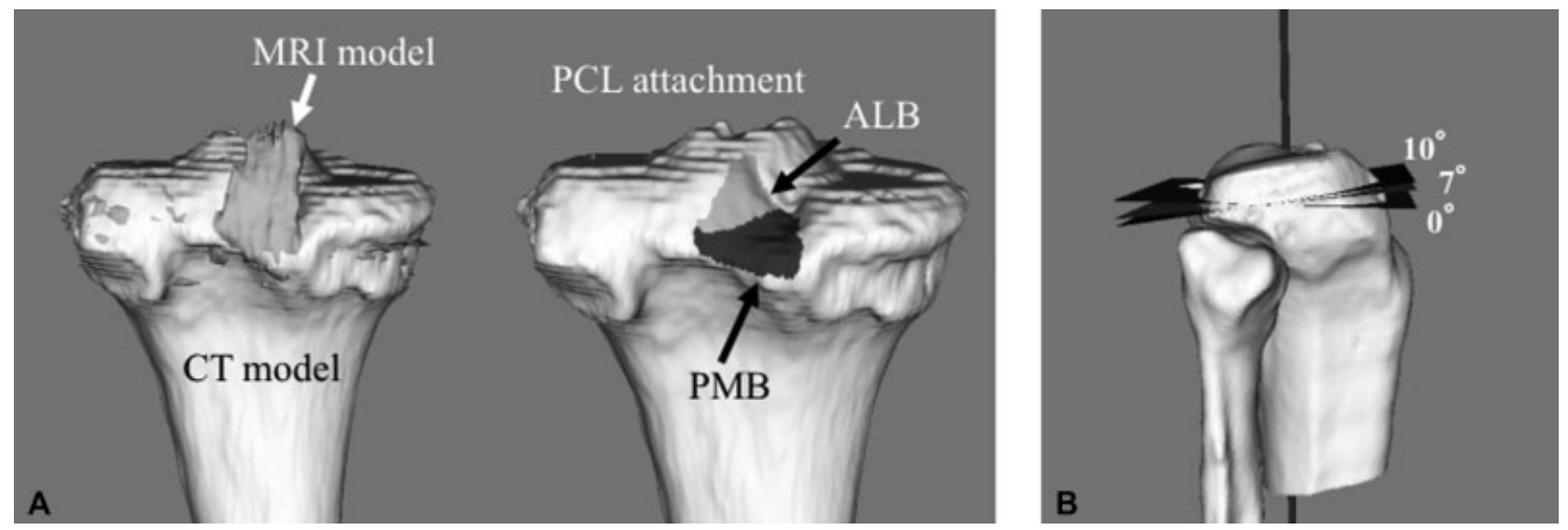

Fig. 1 (A) Method of visualizing the posterior cruciate ligament $(\mathrm{PCL})$ tibial attachment area. Surface registration is performed by superimposing the magnetic resonance imaging (MRI) model of the tibia over the computed tomography (CT) model. Anterolateral bundle (ALB) and posteromedial bundle (PMB) attachment areas are identified on the basis of site morphology. (B) Tibial bone cut. The three-dimensional CT model of the tibia is cut at 8 and $10 \mathrm{~mm}$ distal to the center of the lateral articular surfaces with a posterior tibial slope of 0,7 , or 10 degrees.

posterior tibial slope of 0,7 , or 10 degrees (-Fig. 1B). These parameters were based on the assumed articular cartilage thickness.

\section{Calculation of the Residual Bony Attachment Areas of the $\mathrm{PCL}, \mathrm{ALB}$, and PMB}

Orthopaedics Viewer was used to calculate the PCL, ALB, and PMB attachment areas in the 3D CT model of the tibia before and after tibial bone cut simulation. ${ }^{18}$

\section{Statistical Analysis}

A multiple comparison test (Steel-Dwass test), Student's ttest, and the Spearman rank correlation coefficient were used for statistical analysis, with the significance level set at 0.05. Statistical tests were performed using JMP 13 (SAS Institute Inc., Cary, $\mathrm{NC}$ ).

\section{Results}

The mean PCL attachment area was $220.8 \mathrm{~mm}^{2}$ (range: 168.5$322.7 \mathrm{~mm}^{2}$ ). The mean (range) ALB and PMB attachment areas were $80.3 \mathrm{~mm}^{2}\left(51.9-115.3 \mathrm{~mm}^{2} ; 36.6 \%\right)$ and $140.5 \mathrm{~mm}^{2}$ $\left(97.5-234.8 \mathrm{~mm}^{2} ; 63.4 \%\right)$, respectively (-Table 2 ).

The PCL attachment area correlated with the subject height $\left(r_{s}=0.539, p<0.001\right.$; - Fig. 2A) but not with weight $\left(r_{s}\right.$ $=0.245, p=0.142) \quad$ or $\mathrm{BMI} \quad\left(r_{s}=-0.030, p=0.867\right.$; -Fig. 2B, C).

Table 2 Attachment of the PCL

\begin{tabular}{|l|l|l|}
\hline & Area, $\mathrm{mm}^{2}$ (range) & $\begin{array}{l}\text { Percentage of the } \\
\text { PCL (range) }\end{array}$ \\
\hline PCL & $220.8 \pm 33.9(168.5-322.7)$ & \\
\hline ALB & $80.3 \pm 15.5(51.9-115.3)$ & $36.6 \pm 6(25.3-47.9)$ \\
\hline PMB & $140.5 \pm 28.6(97.5-234.8)$ & $63.4 \pm 6(52.1-74.7)$ \\
\hline
\end{tabular}

Abbreviations: ALB, anterolateral bundle; PCL, posterior cruciate ligament; PMB, posterior medial bundle.
When simulating an 8-mm proximal tibial bone cut with posterior tibial slope angles of 0,7 , and 10 degrees, the ALB tibial attachment area was completely removed in 10 knees (0 degrees: 25\%), 26 knees (7 degrees: 65\%), and 33 knees (10 degrees: $82.5 \%$ ), respectively. For cases in which the ALB tibial attachment area was preserved, the residual ALB tibial attachment areas were $21.8 \%$ (range: 0.3-62.5\%), 9.5\% (range: $0.2-28.5 \%$ ), and $8.1 \%$ (range: $1.5-16.7 \%$ ), respectively.

In the same proximal tibial bone cut simulation, the PMB tibial attachment area was completely removed in only two knees ( 7 degrees in one knee, 10 degrees in one knee). In cases in which the PMB tibial attachment area was preserved, the residual PMB tibial attachment areas were 86.3\% (range: $16.7-100 \%$ ), $64.1 \%$ (range: $19.3-99 \%$ ), and $51 \%$ (range: 7.2-96.4\%), respectively.

When simulating a $10-\mathrm{mm}$ proximal tibial bone cut with posterior tibial slope angles of 0,7 , and 10 degrees, the ALB tibial attachment area was completely removed in 27 knees (0 degrees: 67.5\%), 39 knees (7 degrees: 97.5\%), and 39 knees (10 degrees: $97.5 \%$ ), respectively. For cases in which the ALB tibial attachment area was preserved, the residual ALB tibial attachment areas were 9.6 (range, 0.3-29.9\%), 5.2 , and $0.5 \%$, respectively. In the same proximal tibial bone cut simulation, the PMB tibial attachment area was completely removed in five knees (7 degrees in two knees, 10 degrees in three knees). For the cases in which the PMB tibial attachment area was preserved, the residual PMB tibial attachment areas were $64.3 \%$ (range: $0.1-99.2 \%$ ), $38.1 \%$ (range: $4.1-84.6 \%$ ), and $27 \%$ (range: $0.3-70.5 \%$ ), respectively.

In the comparison of each posterior tilt, significant differences in residual ALB attachment area were identified for a tibial cut of $8 \mathrm{~mm}$ with posterior tibial slope angles of 0 and 7 degrees $(p=0.001)$ and of 0 and 10 degrees $(p<0.001$; -Fig. 3A). Significant differences in residual PMB attachment areas were found for a tibial cut of $8 \mathrm{~mm}$ with posterior tibial slope angles of 0 and 7 degrees $(p<0.001)$ and of 0 and 10 degrees $(p<0.001 ;-$ Fig. 3B $)$. 


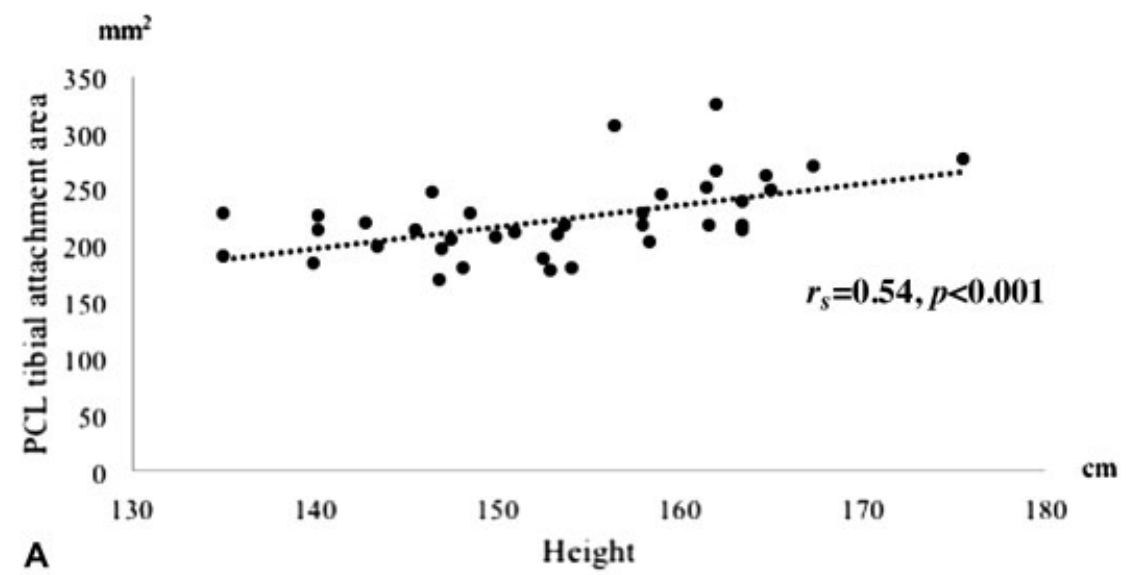

A
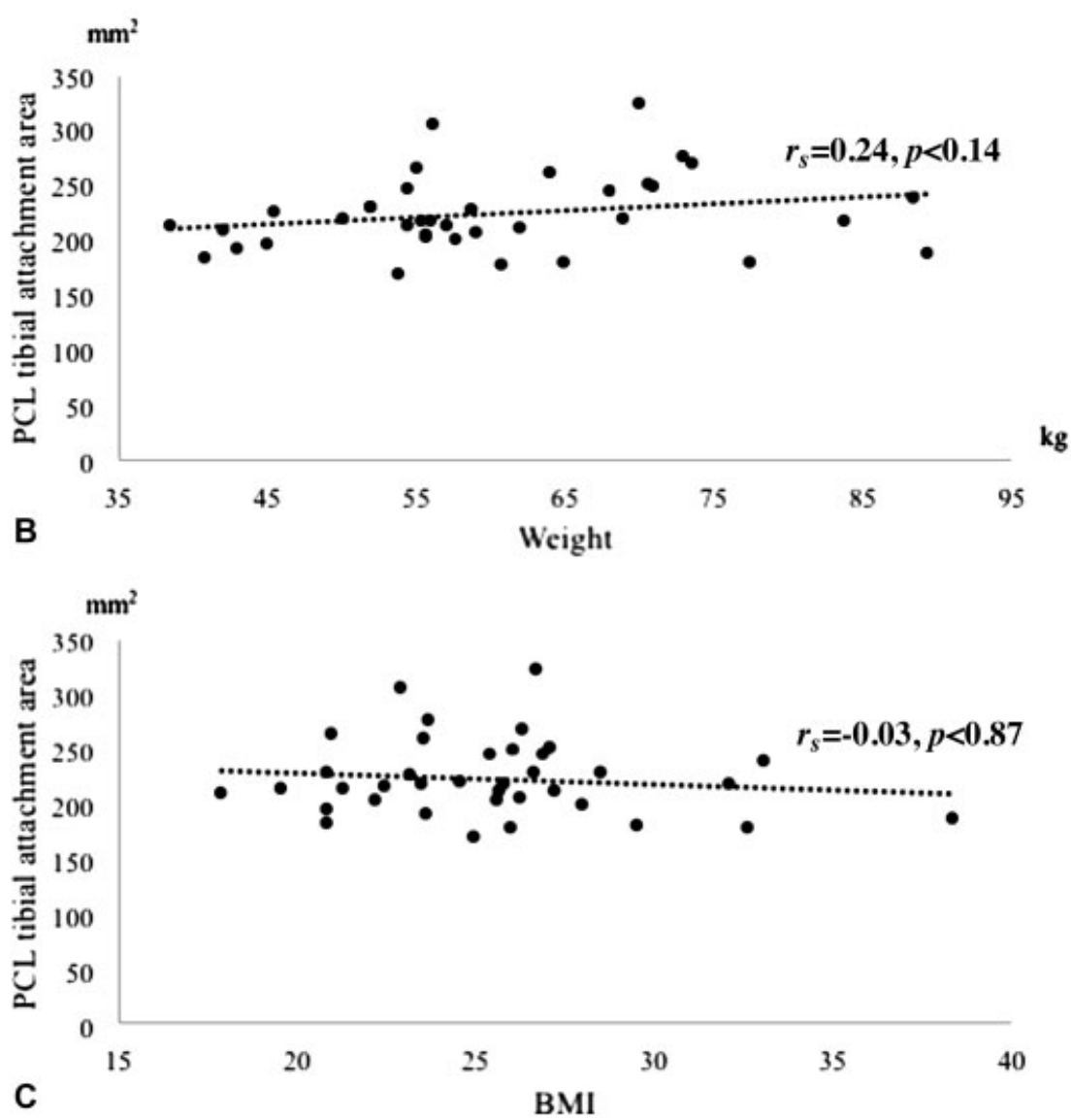

Fig. 2 (A) The posterior cruciate ligament (PCL) attachment area correlates with subject height $\left(r_{s}=0.53 ; p<0.01\right)$. (B,C) The PCL attachment area did not correlate with subject weight or body mass index $\left(r_{s}=0.28, p=0.12 ; r_{s}=0.08, p=0.64\right)$.

\section{Discussion}

In this study, the two bundles of the PCL tibial attachment were examined in simulations of tibial bone cutting during CR-TKA using 3D bone models.

Surface registration enables superimposition of the 3D MRI bone model over the 3D CT bone model. ${ }^{19}$ The 3D MRI bone model visualizes soft tissues, ligaments, and menisci, whereas the 3D CT bone model shows the outlines of the joints. Degeneration of the knee is reportedly less likely to occur at the PCL than at the ACL, and PCL degeneration is not associated with joint deformity. ${ }^{20}$ Furthermore, recent developments in image analysis have enabled clarification of the features of the ligament attachment areas of the PCL, $A L B$, and PMB, and these features were clearly identified in the 3D bone models. ${ }^{12}$

The results of this study concerning the PCL, ALB, and PMB attachment areas were compatible with the results in cadaveric knees, which suggest that the attachment areas were sufficiently reproduced by the 3D bone models. ${ }^{11,12}$

A bone island can be created to preserve the PCL in CRTKA, but the procedure requires consideration of the morphology of the posterior intercondylar tibia, the size of the slot for the PCL attachment area/tibia implant, and the risks 
\%ALB tibial attachment area

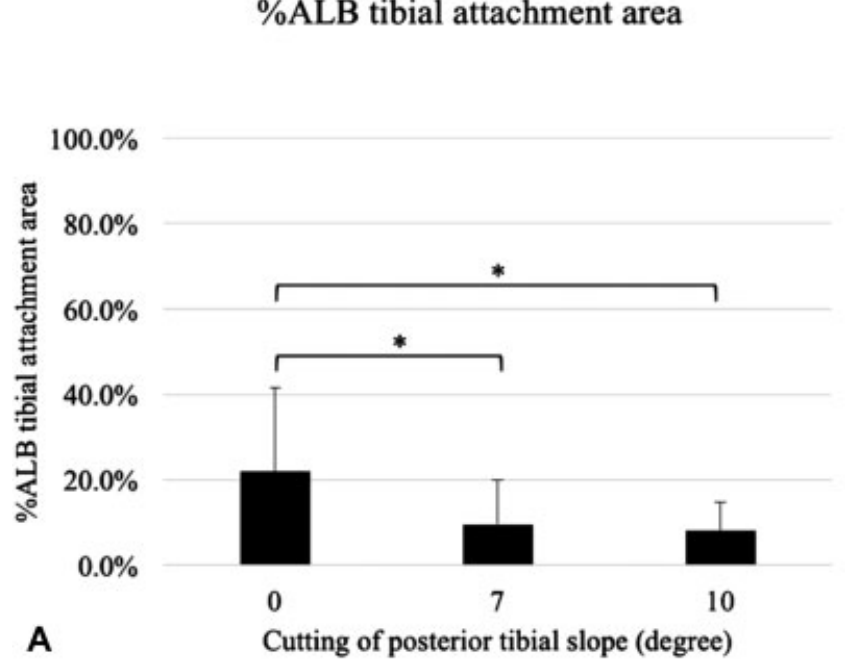

\%PMB tibial attachment area

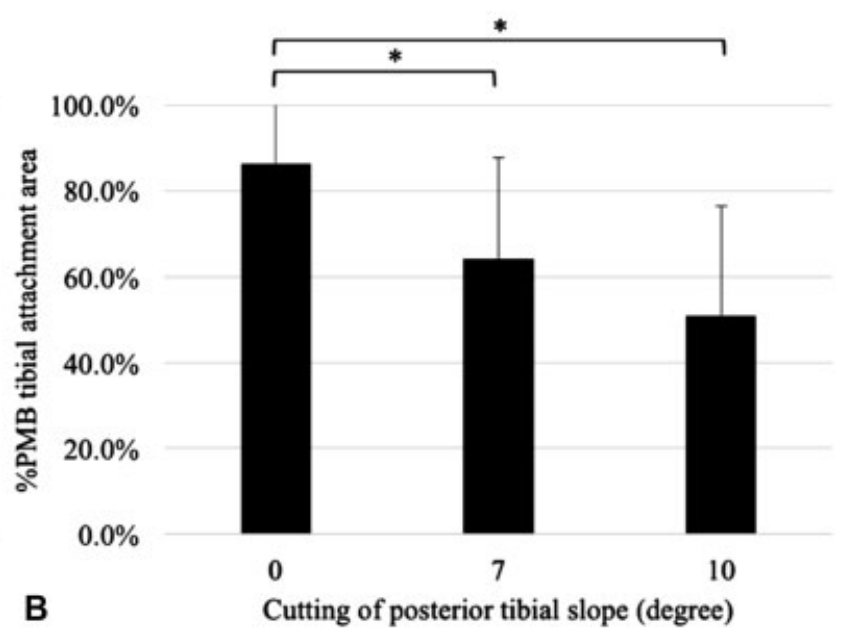

Fig. 3 (A) The residual anterolateral bundle (ALB) attachment area after the tibial cut at $8 \mathrm{~mm}$. The residual ALB attachment area decreases with increasing posterior tibial slope angle, showing significant differences among angles of 0,7 , and 10 degrees $\left({ }^{*} p<0.01\right)$. (B) The residual posteromedial bundle (PMB) attachment after the tibial cut at $8 \mathrm{~mm}$. The residual PMB attachment area also decreases with increasing posterior tibial slope angle, showing significant differences among angles of 0,7 , and 10 degrees $\left({ }^{*} p<0.01\right)$.

of fracture of the bone island. In general, creating a bone island can be difficult in the clinical setting. The recommended posterior tibial slope angle of the tibial implant in CR-TKA is 7 degrees, with an additional bone cut by adding a posterior tibial slope angle if the flexion gap is tight. ${ }^{21,22}$ In this study, bone cutting was simulated without a bone island in the 3D CT model of the tibia at 8 and $10 \mathrm{~mm}$ from the lateral articular surfaces, with posterior tibial slope angles of 0,7 , and 10 degrees. Proximal tibial bone cutting decreased the residual PCL attachment area as the angle increased. With a tibial cut of $8 \mathrm{~mm}$ at a posterior tibial slope angle of 7 degrees, the residual PCL attachment was approximately $50 \%$. The residual attachment area was smaller for the ALB than for the PMB because the attachment area of the ALB was located closer to the articular surfaces of the tibia than that of the PMB. ${ }^{11-14}$ These results suggest that only half of the PCL attachment area was retained after the bone cut simulation. The PMB can therefore be preserved at a posterior tibial slope angle of 7 degrees, unless the PCL attachment is left as a bone island.

One of the important goals of CR-TKA is to preserve the function of each ligament fiber of the PCL. The ALB supports the femoral rotation axis, and the PMB inhibits the posterior translation of the tibia in deep flexion of the knee. ${ }^{23,24}$ This background and the results of this study indicate that ALB dysfunction after partial loss of the ALB attachment area inhibits femoral rotation. Furthermore, paradoxical anterior movement may occur after partial loss of the ALB and PMB attachments. In paradoxical anterior movement and PCL elongation with knee flexion, partial loss of the PCL attachment area may result in dysfunction. ${ }^{8,25}$ However, in our previous studies, bicondylar rollback was observed with deep knee bending after CR-TKA because of the ALB and PMB with a bone island. ${ }^{26,27}$
A major limitation of this study was that residual PCL attachment may depend on the thickness of the cartilage and the amount of the bone cut. This study was limited to an assumed cartilage thickness of $2 \mathrm{~mm}$ and bone cutting in the 3D tibia model of 8 and $10 \mathrm{~mm}$ from the lateral articular surfaces. Furthermore, this study was performed in Asian patients, and thus results may differ for patients from other ethnicities with different bone morphologies.

In conclusion, this study, which used a 3D bone model, found that the PCL attachment area and residual ALB attachment decrease significantly in CR-TKA, with increases to the posterior tibial slope angle and the amount of the tibial bone cut. Complete preservation of the ALB and PMB attachment areas requires selection of surgical procedures and devices such as a procedure to protect the area with a bone island prior to CR-TKA.

Conflict of Interest

None.

\section{References}

1 Mizu-Uchi H, Matsuda S, Miura H, Nabeyama R, Okazaki K, Iwamoto Y. Anteroposterior stability in posterior cruciate ligament-retaining total knee arthroplasty. J Arthroplasty 2006;21 (04):592-598

2 Emodi GJ, Callaghan JJ, Pedersen DR, Brown TD. Posterior cruciate ligament function following total knee arthroplasty: the effect of joint line elevation. Iowa Orthop J 1999;19:82-92

3 Sorger JI, Federle D, Kirk PG, Grood E, Cochran J, Levy M. The posterior cruciate ligament in total knee arthroplasty. J Arthroplasty 1997;12(08):869-879

4 Komistek RD, Mahfouz MR, Bertin KC, Rosenberg A, Kennedy W. In vivo determination of total knee arthroplasty kinematics: a multicenter analysis of an asymmetrical posterior cruciate retaining total knee arthroplasty. J Arthroplasty 2008;23(01):41-50 
5 Swanik CB, Lephart SM, Rubash HE. Proprioception, kinesthesia, and balance after total knee arthroplasty with cruciate-retaining and posterior stabilized prostheses. J Bone Joint Surg Am 2004; 86-A(02):328-334

6 Del Valle ME, Harwin SF, Maestro A, Murcia A, Vega JA. Immunohistochemical analysis of mechanoreceptors in the human posterior cruciate ligament: a demonstration of its proprioceptive role and clinical relevance. J Arthroplasty 1998;13(08):916-922

7 Siston RA, Giori NJ, Goodman SB, Delp SL. Intraoperative passive kinematics of osteoarthritic knees before and after total knee arthroplasty. J Orthop Res 2006;24(08):1607-1614

8 Dennis DA, Komistek RD, Mahfouz MR. In vivo fluoroscopic analysis of fixed-bearing total knee replacements. Clin Orthop Relat Res 2003;(410):114-130

9 Rosenberg AG. Posterior cruciate ligament retention in total knee arthroplasty. In: Insall J, Scott W, eds. Surgery of the Knee. 5th ed. New York, NY: Churchill Livingstone; 2012:1125-1131

10 Amis AA, Gupte CM, Bull AM, Edwards A. Anatomy of the posterior cruciate ligament and the meniscofemoral ligaments. Knee Surg Sports Traumatol Arthrosc 2006;14(03):257-263

11 Anderson CJ, Ziegler CG, Wijdicks CA, Engebretsen L, LaPrade RF. Arthroscopically pertinent anatomy of the anterolateral and posteromedial bundles of the posterior cruciate ligament. J Bone Joint Surg Am 2012;94(21):1936-1945

12 Tajima G, Nozaki M, Iriuchishima T, et al. Morphology of the tibial insertion of the posterior cruciate ligament. J Bone Joint Surg Am 2009;91(04):859-866

13 Takahashi M, Matsubara T, Doi M, Suzuki D, Nagano A. Anatomical study of the femoral and tibial insertions of the anterolateral and posteromedial bundles of human posterior cruciate ligament. Knee Surg Sports Traumatol Arthrosc 2006;14(11):1055-1059

14 Edwards A, Bull AM, Amis AA. The attachments of the fiber bundles of the posterior cruciate ligament: an anatomic study. Arthroscopy 2007;23(03):284-290

15 Matziolis G, Mehlhorn S, Schattat N, et al. How much of the PCL is really preserved during the tibial cut? Knee Surg Sports Traumatol Arthrosc 2012;20(06):1083-1086

16 Aoki M, Shishido T, Takahashi Y, et al. Functional evaluation in cruciate-retaining-type TKA: anatomical relationship between tibial osteotomy level and PCL attachment. Eur J Orthop Surg Traumatol 2014;24(08):1531-1537
17 Kawashima K, Tomita T, Tamaki M, Murase T, Yoshikawa H, Sugamoto K. In vivo three-dimensional motion analysis of osteoarthritic knees. Mod Rheumatol 2013;23(04):646-652

18 Kataoka T, Moritomo H, Omokawa S, Iida A, Murase T, Sugamoto K. Ulnar variance: its relationship to ulnar foveal morphology and forearm kinematics. J Hand Surg Am 2012; 37(04):729-735

19 Lee YS, Seon JK, Shin VI, Kim GH, Jeon M. Anatomical evaluation of CT-MRI combined femoral model. Biomed Eng Online 2008;7:6

20 Aggarwal AK, Goel A, Radotra BD. Predictors of posterior cruciate ligament degeneration in osteoarthritic knees. J Orthop Surg (Hong Kong) 2013;21(01):15-18

21 Parker Vail T, Lang JE, Sikes CV. Surgical techniques and instrumentation in total knee arthroplasty. In: Insall J, Scott W, eds. Surgery of the Knee. 5th ed. New York, NY: Churchill Livingstone; 2012:1042-1099

22 Bellemans J, Robijns F, Duerinckx J, Banks S, Vandenneucker H. The influence of tibial slope on maximal flexion after total knee arthroplasty. Knee Surg Sports Traumatol Arthrosc 2005;13(03): 193-196

23 Wang JH, Kato Y, Ingham SJ, et al. Effects of knee flexion angle and loading conditions on the end-to-end distance of the posterior cruciate ligament: a comparison of the roles of the anterolateral and posteromedial bundles. Am J Sports Med 2014;42(12): 2972-2978

24 Papannagari R, DeFrate LE, Nha KW, et al. Function of posterior cruciate ligament bundles during in vivo knee flexion. Am J Sports Med 2007;35(09):1507-1512

25 Yue B, Varadarajan KM, Rubash HE, Li G. In vivo function of posterior cruciate ligament before and after posterior cruciate ligament-retaining total knee arthroplasty. Int Orthop 2012;36 (07):1387-1392

26 Horiuchi H, Akizuki S, Tomita T, Sugamoto K, Yamazaki T, Shimizu $\mathrm{N}$. In vivo kinematic analysis of cruciate-retaining total knee arthroplasty during weight-bearing and non-weight-bearing deep knee bending. J Arthroplasty 2012;27(06):1196-1202

27 Fujimoto E, Sasashige Y, Tomita T, Iwamoto K, Masuda Y, Hisatome T. Significant effect of the posterior tibial slope on the weight-bearing, midflexion in vivo kinematics after cruciateretaining total knee arthroplasty. J Arthroplasty 2014;29(12): 2324-2330 